\title{
Optical lattice quantum Hall effect
}

\author{
Rebecca N. Palmer ${ }^{1,2}$, Alexander Klein ${ }^{1,3}$, and Dieter Jaksch ${ }^{1,3}$ \\ 1 Clarendon Laboratory, University of Oxford, Parks Road, Oxford OX1 3PU, United Kingdom \\ ${ }^{2}$ School of Physics and Astronomy, University of Leeds, Leeds LS2 9JT, United Kingdom \\ 3 Keble College, Parks Road, Oxford OX1 3PG, United Kingdom
}

(Dated: October 23, 2018)

\begin{abstract}
We explore the behavior of interacting bosonic atoms in an optical lattice subject to a large artificial magnetic field. We extend earlier investigations of this system where the number of magnetic flux quanta per unit cell $\alpha$ is close to a simple rational number [Phys. Rev. Lett. 96, 180407 (2006)]. Interesting topological states such as the Laughlin and Read-Rezayi states can occur even if the atoms experience a weak trapping potential in one direction. An explicit numerical calculation near $\alpha=1 / 2$ shows that the system exhibits a striped vortex lattice phase of one species, which is analogous to the behavior of a two-species system for small $\alpha$. We also investigate methods to probe the encountered states. These include spatial correlation functions and the measurement of noise correlations in time-of-flight expanded atomic clouds. Characteristic differences arise which allow for an identification of the respective quantum Hall states. We furthermore discuss that a counterintuitive flow of the Hall current occurs for certain values of $\alpha$.
\end{abstract}

PACS numbers: 37.10.Jk,03.75.Lm,73.43.-f

\section{INTRODUCTION}

Since their discovery, the integer [1] and fractional quantum Hall effects [2, 3] have attracted much attention on the theoretical as well as on the experimental side. They were first observed in semi-conductor samples which confine the electrons in a quasi two-dimensional layer and are characterized by the ratio $\nu$ of particles to magnetic flux quanta penetrating the layer. For the integer quantum Hall effect, this ratio is an integer number, whereas the fractional quantum Hall effect exhibits a simple rational (non-integer) $\nu$ and has interparticle interactions as an essential component. Some of the excitations of the fractional quantum Hall effect are promising candidates for topological quantum computing [4], since they are separated from the ground state by an energy gap and they might exhibit fractional, anyonic statistics [5, 6, 7].

Despite the work which has been put into this field, the mechanisms which lead to the Hall effects are not completely understood [7, 8]. Especially theoretical investigations are hampered by the complexity of the system: A many-body quantum description is necessary to capture the relevant effects such as the strong correlation of the electrons, which means that the calculational effort scales exponentially with the number of particles to be described. To gain insight into the behavior of the quantum Hall effects it is therefore worthwhile to investigate whether these phenomena occur in alternative systems as well.

Ultracold atoms in optical lattices are a system where the quantum Hall effect can indeed be observed. As some of the present authors have shown in an earlier publication [9], for a suitable choice of the external parameters various topological states occur in these systems such as the Laughlin state [10] or the Read Rezayi states [11]. These states have been derived by developing a model that is valid near simple rational numbers of magnetic flux quanta per lattice cell, $\alpha$. We showed that the occurring states can be distinguished by shot noise and Hall current measurements, where in the latter case unexpected sign changes can occur.

In the present paper we extend these investigations. We will give details of how to expand the Hamiltonian describing cold bosonic atoms in an optical lattice subject to an artificial magnetic field for the case that $\alpha$ is close to a simple rational $\alpha_{c}=l / n$ with $l$ and $n$ small integers. We will also give more insight into how to distinguish the occurring states by noise correlation or Hall current measurements. In addition to these extensions, we focus on other phenomena occurring in our setup. By using a Gutzwiller ansatz we demonstrate that for $\alpha$ close to $\alpha_{c}=1 / 2$ and large filling fractions a two-component-like striped vortex state occurs which shows that the analytical description derived in [9] is meaningful. The presence of the magnetic field leads to an additional small scale structure for values of $\alpha$ close to $\alpha_{c}$, which can be made visible in time-of-flight expansions as we will illustrate for $\alpha_{c}=1 / 2$.

The applicability of our scheme is ensured by the experimental and theoretical successes in the field of optical lattices during the last couple of years. Theoretical investigations have shown that a whole wealth of models can be simulated [12], such as the Hubbard Hamiltonian for bosons, fermions, or mixtures [13, 14], spin-spin interactions [15, 16], high-temperature superconductivity [17, 18], or the formation of polarons [19, 20, 21]. Theoretical investigations show that these models exhibit rich phase diagrams which should be accessible with current experimental techniques [16, 22, 23, 24, 25]. The necessary low temperatures can be achieved by appropriate cooling methods [26, 27, 28, 29]. It is also possible to implement artificial magnetic fields in this setup, for example by rotating the lattice [30, 31, 32, 33]. This comes, however, with the disadvantage that a centrifugal term 
occurs, which has to be balanced by an external trapping potential. To circumvent this problem alternative schemes have been developed, exploiting Raman assisted hopping [34, 35], oscillating quadrupole fields 36], or laser beams with orbital angular momentum [37, 38, 39]. Some of these schemes even exhibit non-Abelian gauge potentials [40, 41], allowing for the investigation of new phenomena in the integer quantum Hall effect [42] or the observation of the non-Abelian Aharanov-Bohm effect [43].

In the presence of a lattice, the strength of the artificial magnetic field is suitably characterized by the number of effective magnetic flux quanta per unit cell, $\alpha$, which for a conventional semiconductor setup is typically very small, $\alpha \ll 1$. In the optical lattice setup, however, it is possible to achieve values close to $\alpha=1 / 2$, a regime which is almost impossible to achieve in the condensed matter case due to the required huge magnetic fields. This allows for the experimental investigation of so far unobserved phenomena such as the Hofstadter butterfly [34, 44, 45, 46], a fractal energy spectrum originally predicted for electrons subject to a large magnetic field. It is also worthwhile to investigate the energy gap of anyonic excitations near $\alpha=1 / 2$. If it is sufficiently large, it would make topological quantum computing more feasible since thermally excited anyons are the major source of errors in these schemes [4].

There have been previous investigations of ultracold atoms in optical lattices subject to large artificial magnetic fields or rotation. For instance, in [47] a rotation scheme of an optical lattice was proposed which leads to quantum Hall and spin liquid properties of a spin-1 boson cluster. The dependence of the state on the rotation frequency has been investigated in Ref. [48], where it was shown that one can change the state adiabatically from a Gaussian to a Laughlin state. Transport properties of one and two particles in a rotating lattice have been investigated in Ref. [49] using a Kubo formalism. Recently, the influence of boundary conditions on the Hall states was investigated and Chern numbers were employed to characterize the signature of the topological states [50, 51]. These authors also discussed that the quantum Hall states can be made more stable and the energy gap to excitations can be increased by using atoms with a strong dipole-dipole interaction such as chromium [50].

An experimental implementation of the proposed methods and the observation of the predicted effects should be possible in the near future [14]. The creation of fast rotating traps has already been demonstrated in Refs. [52, 53], which have allowed one to investigate effects such as vortex pinning [54]. Loading an optical lattice with a well-defined number of atoms, thereby allowing one to tune $\nu$ independently from the strength of the artificial magnetic field, has been achieved by exploiting spin oscillations [55, [56, [57], and the number statistics of ultracold atom systems has been examined in [58, 59]. Also, measurement schemes have been im- plemented which allow for an investigation of second order correlation functions such as noise correlations [60], thereby revealing additional quantum properties of the states.

Our paper is organized as follows. In Sec. III we introduce the Hamiltonian which describes the atoms in the optical lattice subject to an artificial magnetic field in Landau gauge. For weak magnetic fields, a continuum approximation is applied as shown in Sec. III]. We extend our investigations in Sec. IV to higher artificial magnetic fields, such that $\alpha$ is close to simple rational values where the system is well-described by multi-component continuous wave functions. In Sec. $\mathrm{V}$ we present how the different quantum Hall states can be distinguished from each other. The used methods include time-of-flight expansions, measuring noise correlations in the time-of-flight expanded clouds, and observing the occurring Hall mass current. We conclude in Sec. VI.

\section{HAMILTONIAN}

Throughout this work we assume a two-dimensional optical lattice [13, 14], which can be experimentally achieved for example by using a three-dimensional lattice with the hopping into one direction strongly suppressed [61]. The atoms are then confined in two-dimensional planes, of which we consider only one in the following. The artificial magnetic field term is created using one of the several methods discussed in the Introduction [34, 35, 36, 37, 38, 39]. We furthermore assume that the total potential energy experienced by the atoms is given by $V(p, q)$, where $p, q$ label the lattice sites in the $x$ - and $y$-directions, respectively. In Landau gauge, which is more convenient for our purposes, the Hamiltonian describing the atoms is given by $[9,34,36]$

$$
\begin{aligned}
\hat{H}= & -\sum_{p, q}\left(J_{x} \mathrm{e}^{2 \pi \mathrm{i} \alpha q} \hat{a}_{p, q}^{\dagger} \hat{a}_{p-1, q}+J_{y} \hat{a}_{p, q}^{\dagger} \hat{a}_{p, q-1}+\text { H.c. }\right) \\
& +V(p, q) \hat{a}_{p, q}^{\dagger} \hat{a}_{p, q}+\frac{U}{2} \hat{a}_{p, q}^{\dagger} \hat{a}_{p, q}^{\dagger} \hat{a}_{p, q} \hat{a}_{p, q},
\end{aligned}
$$

where the magnetic field strength is parameterized by the number of flux quanta per lattice cell $\alpha$. The operator $\hat{a}_{p, q}^{\dagger}$ creates an atom in lattice site $(p, q), J_{x}$ and $J_{y}$ are the hopping constants in the $x$ and $y$ directions, respectively, and $U$ describes the strength of the on-site interaction between the atoms. Due to the artificial magnetic field terms, the atoms acquire a phase $2 \pi \alpha$ when they hop around a lattice cell once. Since the value of $\alpha$ is only defined modulus one, we may restrict it to $0 \leq \alpha<1$.

It is instructive to compare our setup to other systems in which the quantum Hall effect can be observed, such as a semiconductor structure or a rotating ultracold atomic gas without an optical lattice. The main characteristics and typical values are shown in Table [. Due to the small lattice spacing $d \sim 10^{-10} \mathrm{~m}$ in the original solid 
state semiconductor systems, for instance, $\mathrm{Si}$ or GaAs, typically only small values $\alpha=e B d^{2} / 2 \hbar \pi \sim 10^{-4}$ can be achieved with available magnetic fields $B$. Here, $e$ is the charge of the electron. Although this can be overcome by using superlattice structures [45, 62], defects or impurities are inevitable in a real crystal. In contrast, the optical lattice setup allows for a virtually defect-free implementation of the quantum Hall Hamiltonian, and values up to $\alpha \sim 1$ can be achieved [34, 35, 36, 50]. Comparing the cyclotron frequency $\Omega=\pi \alpha \hbar / \bar{m} d^{2}$ (for a definition of the effective mass $\bar{m}$ in the lattice setup see the next section) to the temperatures which can be achieved in the respective realizations shows that similar regimes can be realized with all three methods when appropriate cooling techniques are exploited in the atom setup [26, 27, 28, 29]. Low ratios of temperature over magnetic field are important to avoid thermal excitations which would spoil the applicability of the system for topological quantum computation [7].

In contrast to the semiconductor scheme, the two atomic realizations provide more opportunities for tunability and probing the states. Both atom setups allow for additional measurements to be carried out, such as time-of-flight expansions and measuring second order correlation functions [60, 63]. The lattice setup furthermore enables an easy tuning of the system parameters such as the hopping or the interaction between the atoms [14]. In a standard optical lattice setup, this interaction is given by a contact term $\delta(r)$. By using certain atomic species such as chromium one can also implement dipole-dipole interactions which might stabilize the quantum Hall states [50]. It is also possible to extend the contact interaction by immersing the optical lattice into a Bose-Einstein condensate, where an additional off-site interaction occurs [19, 20, 64].

Compared to the rotating setup, in a lattice there exist methods which do not involve a centrifugal term that has to be balanced by an additional external potential. Although experiments with rotating traps already exist [52, 53, 54], reaching the quantum Hall regime is challenging due to the need of balancing the centrifugal force.

\section{SMALL $\alpha$ LIMIT: CONTINUUM APPROXIMATION}

In earlier publications [9, 36], it was shown that for small values of $\alpha$ the influence of the optical lattice is negligible and we can approximate the lattice gas by a continuous wave function. In the present section, we give a more detailed account of this analysis. We first consider a single particle in the optical lattice described by the discrete wave function $|\psi\rangle=\sum_{p, q} \psi(p, q) \hat{a}_{p, q}^{\dagger}|0\rangle$, which obeys the normalization condition $\sum_{p, q}|\psi(p, q)|^{2}=1$.
The Hamiltonian Eq. (11) acts on this wave function as

$$
\begin{aligned}
\hat{H}|\psi\rangle=\sum_{p, q} & \left\{-J_{x}\left[\mathrm{e}^{2 \pi \mathrm{i} \alpha q} \psi(p-1, q)+\mathrm{e}^{-2 \pi \mathrm{i} \alpha q} \psi(p+1, q)\right]\right. \\
& -J_{y}[\psi(p, q-1)+\psi(p, q+1)] \\
& +V(p, q) \psi(p, q)\} a_{p, q}^{\dagger}|0\rangle .
\end{aligned}
$$

For $\alpha \ll 1$ and a weak trapping potential $V(p, q)$, the wave function varies only slowly from one lattice site to the neighboring ones, and we can approximate the state by a continuous wave function $\phi(x, y)$, where $\psi(p, q)=d \phi(p d, q d)$ with $d$ the lattice spacing and $\int|\phi(x, y)|^{2} \mathrm{~d} x \mathrm{~d} y=1$. The dynamics of this wave function is governed by the Hamiltonian

$$
\begin{aligned}
H_{0}= & -J_{x}\left[2-\frac{d^{2}}{\hbar^{2}}\left(\mathrm{i} \hbar \frac{\partial}{\partial x}-\frac{2 \pi \alpha \hbar y}{d^{2}}\right)^{2}\right] \\
& -J_{y}\left[2-\frac{d^{2}}{\hbar^{2}}\left(\mathrm{i} \hbar \frac{\partial}{\partial y}\right)^{2}\right]+V(x, y) .
\end{aligned}
$$

This Hamiltonian can be transformed into a more familiar form by defining the effective masses $m_{x, y}=$ $\hbar^{2} / 2 J_{x, y} d^{2}, \bar{m}=\sqrt{m_{x} m_{y}}$, and the cyclotron frequency $\Omega=\pi \alpha \hbar / \bar{m} d^{2}$. After discarding a constant energy term we get

$$
H_{0}=\frac{1}{2 m_{x}}\left(\mathrm{i} \hbar \frac{\partial}{\partial x}-2 \bar{m} \Omega y\right)^{2}-\frac{\hbar^{2}}{2 m_{y}} \frac{\partial^{2}}{\partial y^{2}}+V(x, y) .
$$

This is the familiar single particle quantum Hall Hamiltonian with an artificial "electric" field potential $V(x, y)$ and anisotropic mass, which can be redefined into an anisotropic magnetic length. For deriving the energy levels we assume that the potential $V(x, y)$ is constant in the $x$ direction and forms a harmonic potential in the $y$ direction, $V(x, y)=m_{y} \omega^{2} y^{2} / 2$. The Hamiltonian is then translational invariant in the $x$ direction, which justifies the ansatz $\phi(x, y)=\exp (-\mathrm{i} K x) F(y)$. The Hamiltonian $H_{0}$ acts on this wave function as

$$
H_{0} \phi=\left[-\frac{\hbar^{2}}{2 m_{y}} \frac{\partial^{2}}{\partial y^{2}}+\frac{1}{2} m_{y} \omega_{\mathrm{eff}}^{2}\left(y-y_{c}\right)^{2}+\frac{\omega^{2} \hbar^{2} K^{2}}{2 m_{x} \omega_{\mathrm{eff}}^{2}}\right] \phi,
$$

where $\omega_{\text {eff }}=\sqrt{4 \Omega^{2}+\omega^{2}}$, and $y_{c}=2 \Omega \hbar K / \omega_{\text {eff }}^{2} \bar{m}$. This expression describes a displaced harmonic oscillator with an energy offset depending on $K$. For $\omega=0$ we retain the usual Landau levels, whereas for $\omega \neq 0$ the degeneracy within one Landau level is lifted and the energies are given by

$$
E=\left(n_{\mathrm{LL}}+\frac{1}{2}\right) \hbar \omega_{\mathrm{eff}}+\frac{\omega^{2} \hbar^{2} K^{2}}{2 m_{x} \omega_{\mathrm{eff}}^{2}}
$$

where $n_{\mathrm{LL}} \geq 0$ is an integer.

The continuum approximation can be extended to the case of more than one particle in the lattice, provided 


\begin{tabular}{|c|c|c|c|}
\hline System & Electrons (GaAs) & Rotating gas $(\mathrm{Rb})$ & Lattice $(\mathrm{Rb})$ \\
\hline Particles & Electrons & Atoms & Atoms \\
\hline Statistics & Fermi & Bose & Bose \\
\hline Interaction potential & $1 / r$ & $\delta(r)$ & $\delta(r)$ \\
\hline Confinement & Sharp edges & Smooth trap & Smooth trap \\
\hline \multicolumn{4}{|l|}{ Physical parameters } \\
\hline System diameter & $\sim 10^{-2} \mathrm{~m}$ & $10^{-5}-10^{-4} \mathrm{~m}$ & $10^{-5}-10^{-4} \mathrm{~m}$ \\
\hline Number of particles $N$ & $\sim 10^{11}$ & $10^{5}-10^{6}$ & $\sim 10^{4}$ \\
\hline 2D number density $\varrho$ & $1-2 \times 10^{15} \mathrm{~m}^{-2}$ & $\sim 10^{12} \mathrm{~m}^{-2}$ & $10^{10}-10^{11} \mathrm{~m}^{-2}$ \\
\hline Cyclotron frequency $(\mathrm{CF}) \Omega$ & $\sim 10^{14} \mathrm{~Hz}$ & $10^{2}-10^{3} \mathrm{~Hz}$ & $\sim 10^{3} \mathrm{~Hz}$ \\
\hline Free mass $m_{0}$ & $9 \times 10^{-31} \mathrm{~kg}$ & $1.5 \times 10^{-25} \mathrm{~kg}$ & $1.5 \times 10^{-25} \mathrm{~kg}$ \\
\hline Effective mass $m$ & $6 \times 10^{-32} \mathrm{~kg}$ & $1.5 \times 10^{-25} \mathrm{~kg}$ & $10^{-24}-10^{-23} \mathrm{~kg}$ \\
\hline Lattice spacing $d$ & $2 \times 10^{-10} \mathrm{~m}$ & $\mathrm{n} / \mathrm{a}$ & $\sim 10^{-6} \mathrm{~m}$ \\
\hline Temperature $T$ & $0.1-0.3 \mathrm{~K}$ & $\sim 10^{-9} \mathrm{~K}$ & $\sim 10^{-11} \mathrm{~K}$ \\
\hline \multicolumn{4}{|l|}{ Dimensionless parameters } \\
\hline$\alpha$ (flux quanta/unit cell) & $10^{-4}$ & $\mathrm{n} / \mathrm{a}$ & $0.1-0.5$ \\
\hline$\nu$ (particles/flux quanta) & $0.2-10$ & $10^{3}-10^{4}$ & $1-10$ \\
\hline$\varrho u / \hbar \Omega$ (interaction/CF) & $\mathrm{n} / \mathrm{a}$ & $0.1-1$ & $\sim 0.1$ \\
\hline$k_{B} T / \hbar \Omega$ (temperature/CF) & $10^{-3}$ & $10^{-1}-1$ & $\sim 10^{-3}$ \\
\hline Internal states & spin or bilayer & hyperfine & hyperfine \\
\hline \multicolumn{4}{|l|}{ Tunability } \\
\hline$\Omega, \varrho, T$ & yes & yes & yes \\
\hline$u m$ & no & yes & yes \\
\hline$m_{x} / m_{y}$ & no & no & yes \\
\hline
\end{tabular}

TABLE I: Comparison of quantum Hall systems. The regime of interest is $\nu \sim 1$, and $\hbar \Omega \gg \varrho u \gg k_{\mathrm{B}} T$. The values for the BEC are from current experiments [52, 53], which are still slightly outside this regime. The low temperatures for the lattice system can be achieved by using, for instance, the methods in Refs. [26, 27, 28, 29]. For the lattice, the data shown is for a single plane only. If the two-dimensional plane is realized using a three-dimensional lattice with the hopping in one direction strongly suppressed, the quantum Hall effect occurs independently in each plane and multiple planes can be used to increase the signal strength without increasing $\nu$.

that the wave function still varies slowly over the distance $d$ between two lattice sites. To achieve this, the average distance between the particles needs to be large compared to $d$, which restricts the particle density to $\varrho \ll 1 / d^{2}$. In this case, the continuum Hamiltonian is given by

$$
H=\sum_{j} H_{0}\left(x_{j}, y_{j}\right)+\frac{u}{2} \sum_{i, j} \delta\left(x_{i}-x_{j}\right) \delta\left(y_{i}-y_{j}\right),
$$

where $u=U d^{2}$. This Hamiltonian describes the quantum Hall effect with a contact interaction term, in contrast to the typically screened $1 / r$ interaction of the Coulomb potential for electrons in solids. In analogy to the solid state fractional quantum Hall effect we define the filling factor $\nu=\hbar \varrho \pi / \bar{m} \Omega=\varrho d^{2} / \alpha$. Since, in this section, we are interested in the limits $\alpha \ll 1$ and $\varrho \ll 1 / d^{2}$ we will hence find the same states as in a continuum bosonic quantum Hall system, for example, the $\nu=1 / 2$ Laughlin state, the $\nu=1,3 / 2, \ldots$ Read-Rezayi states, or a vortex lattice [9, 11, 65].

The strength of the interparticle interaction $u$ plays a crucial role if the setup is to be used for topological quantum computing. For this, anyonic quasiholes are created and they are moved around each other using focussed lasers, thereby inducing qubit operations [31]. However, for the Read-Rezayi (non-Abelian anyon) states to appear the interaction must be weak compared to the spacing between the Landau levels given by $2 \hbar \Omega$, to avoid excitations to higher levels. This reduces the quasiparticlequasihole pair creation gap $\Delta_{g} \sim u m \Omega / 2 \pi \hbar$, where we assume $m \equiv \bar{m}=m_{x}=m_{y}$. As thermally created anyons moving around the computational anyons are a source of error, such computation would require low temperatures $T \ll u m \Omega / 2 \pi \hbar k_{\mathrm{B}} \ll 2 \hbar \Omega / k_{\mathrm{B}}$, which for typical experimental setups are on the order of a few $10 \mathrm{nK}$. These temperatures might be reached using the methods of [26, 27, 28, 29]. Alternatively, it has been suggested that non-Abelian field quantum Hall states [12, 40] may offer non-Abelian excitations in the lowest density state, allowing a strong interaction to be used to increase the gap to $\sim \hbar \Omega$, but this has yet to be confirmed. 


\section{NEAR SIMPLE RATIONAL $\alpha$ : MULTI-COMPONENT WAVEFUNCTIONS}

We will now relax the condition of small $\alpha$ and investigate the properties of the quantum gas for an $\alpha$ close to simple rational values $\alpha \approx l / n$, where $l$ and $n$ are small integers. The wave function can then be approximated by a set of $n$ smooth, slowly varying functions which correspond to $n$ different components of the gas. These components should not be confused with, for instance, different internal states of the atoms. They rather correspond to different small scale structures of the gas. Based on previous work [9], we will give details on how to derive these expressions and consider some special cases. We especially calculate the ground state wave function for a set of representative parameters numerically, which confirms that for $\alpha$ close to $1 / 2$ the two-component description is meaningful. For simplicity, in the following we will consider the case of isotropic hopping, that is, $J_{x}=J_{y} \equiv J$, leading to effective masses $m_{x}=m_{y}=\bar{m} \equiv m$.

\section{A. Single particle states}

As discussed in [9], numerical calculations for simple $\alpha=l / n$ and weak external potentials $V$ suggest that the single particle ground state functions exhibit an $n$ site periodic pattern superimposed on a smooth largescale variation. This motivates the representation $\psi(n p+$ $i, n q+j)=d \sum_{k} \chi_{k}(d(n p+i), d(n q+j)) \mathbf{v}_{i j}^{(k)}$, where $\chi_{k}$ is a continuous, slowly varying function and $\mathbf{v}^{(k)}$ an $n \times n$ matrix describing the small scale structure of the atomic gas. We find by expansion about $\alpha_{c} \equiv l / n$ (see Appendix A for details) that there are $n$ degenerate matrices $\mathbf{v}^{(k)}$ of the form $\mathbf{v}_{p q}^{(k)}=e^{2 \pi i p k / n} \mathbf{v}_{q+k}$, where $\mathbf{v}$ is a fixed $n$ component vector for each $l, n$ and the subscript $q+k$ wraps around $\bmod n$. Furthermore, the $\chi_{k}$ obey the condition

$$
\begin{aligned}
-\frac{C}{2 m} & \frac{\hbar^{2} \partial^{2} \chi_{k}}{\partial y^{2}}+\frac{C}{2 m}\left(2 m \tilde{\Omega} y-i \hbar \frac{\partial}{\partial x}\right)^{2} \chi_{k}+V(x, y) \chi_{k} \\
& =\left(E-\frac{E_{0}}{m d^{2}}\right) \chi_{k}
\end{aligned}
$$

where $\tilde{\Omega} \equiv \hbar\left(\alpha-\alpha_{c}\right) \pi /\left(m d^{2}\right)$ and $C, E_{0}$ depend only on $l, n$. This formula reduces to Eq. (4) for $\alpha_{c}=0 / 1$, and agrees well with numerical calculations near other simple $\alpha_{c}$, especially $\alpha_{c}=1 / 2[9]$.

For this procedure to be consistent, the length scale $l_{\chi}$ over which $\chi_{k}$ varies, which for a harmonic trap $V(x, y)=$ $\frac{1}{2} m \omega^{2} y^{2}$ is given by $l_{\chi}=\left(\hbar^{2} C /\left(4 C m^{2} \tilde{\Omega}^{2}+m^{2} \omega^{2}\right)\right)^{1 / 4}$, must be large compared to the "small scale" periodicity $n d$, but small enough that $2 m \tilde{\Omega} l_{\chi} d / \hbar \ll 1$ (cf. Appendix (A), that is

$$
\frac{1}{n d} \gg \frac{1}{l_{\chi}} \gg \frac{2 m d \tilde{\Omega}}{\hbar}
$$

or equivalently

$$
\frac{1}{n} \gg\left[\frac{\pi^{2}}{C}\left(4 C\left(\alpha-\alpha_{c}\right)^{2}+\beta^{2}\right)\right]^{\frac{1}{4}} \gg 2 \pi\left(\alpha-\alpha_{c}\right) .
$$

Here $\beta=m d^{2} \omega / \hbar \pi$ is the dimensionless trap strength. The first inequality shows that the range over which the multi-component ansatz is valid gets narrower for larger denominators $n$ of $\alpha_{c}$. We also see that the presence of the trap is important to ensure the validity of the second inequality. A stronger trap makes it easier to fulfill the second condition, however, a too strong trap will ultimately lead to a contradiction with the first condition. Indeed, in Ref. [9] it was shown that for small $n$ the above wave functions compare very well with numerical exact calculations and overlaps of more than $99 \%$ can be achieved for $\alpha \approx \alpha_{c}$ and appropriately strong traps.

\section{B. Interacting particles}

Eq. (8) is extended to the many-particle case as

$$
\begin{array}{rl}
H \approx \int \mathrm{d} & \mathrm{d} y \sum_{k} \chi_{k}^{\dagger}(x, y)\left\{-\frac{C \hbar^{2}}{2 m} \frac{\partial^{2}}{\partial y^{2}}\right. \\
+ & \left.\frac{C}{2 m}\left(2 m \tilde{\Omega} y-i \hbar \frac{\partial}{\partial x}\right)^{2}+V(x, y)\right\} \chi_{k}(x, y) \\
+ & u \sum_{k_{1}, k_{2}, k_{3}, k_{4}} G_{k_{1}, k_{2}, k_{3}, k_{4}} \chi_{k_{1}}^{\dagger}(x, y) \chi_{k_{2}}^{\dagger}(x, y) \\
& \times \chi_{k_{3}}(x, y) \chi_{k_{4}}(x, y)+\frac{E_{0}}{m d^{2}},
\end{array}
$$

where $G_{k_{1}, k_{2}, k_{3}, k_{4}} \equiv \sum_{j} \mathbf{v}_{j+k_{1}} \mathbf{v}_{j+k_{2}} \mathbf{v}_{j+k_{3}} \mathbf{v}_{j+k_{4}} / n$ if $k_{1}+$ $k_{2} \equiv k_{3}+k_{4} \bmod n$, and 0 otherwise. The conservation $\bmod n$ is due to the $x$ quasimomentum $2 \pi k / n d$ carried by $\mathbf{v}^{(k)}$. To give an example, for $\alpha_{c}=1 / 2$ we find $G_{1111}=$ $G_{2222}=3 / 2$ and $G_{1212}=G_{2121}=G_{1122}=G_{2211}=1 / 2$. Particles with the same $k$ interact more strongly because their $\mathbf{v}^{(k)}$ are peaked on the same sites.

For $\alpha_{c}=1 / 2$, a change of basis from $\chi_{1,2}$ to $\chi_{ \pm}=$ $\chi_{1} \pm \mathrm{i} \chi_{2}$ makes this effective Hamiltonian analogous to a bilayer fractional quantum Hall system [66], with $\chi_{ \pm}$ being the two "layers". However, the interaction ratio is 1:2 with the "interlayer" interaction being the stronger one, while it is equal or weaker in most other realizations of multicomponent fractional quantum Hall states.

\section{Some special states}

For the case that $\alpha_{c}=1 / 2$ the system is equivalent to a two-component gas as argued in the previous section. It is well-established that for an interacting two-component gas the lowest Landau level state with highest density and zero interaction energy is the so-called 221 state [67] 
defined by

$$
\begin{aligned}
& \phi_{221}\left(z_{1}, \ldots, z_{N / 2}, w_{1}, \ldots, w_{N / 2}\right) \\
& =\left[\prod_{i>j}\left(z_{i}-z_{j}\right)^{2}\right]\left[\prod_{i>j}\left(w_{i}-w_{j}\right)^{2}\right]\left[\prod_{i, j}\left(z_{i}-w_{j}\right)\right] \\
& \quad \times \exp \left(-\sum_{i}\left|z_{i}\right|^{2} / 4+\left|w_{i}\right|^{2} / 4\right),
\end{aligned}
$$

where there are $N / 2$ particles in one component with coordinates $z=(x+\mathrm{i} y) / r_{0}$ and $N / 2$ in the other component with coordinates $w=(x+\mathrm{i} y) / r_{0}$, and $r_{0}=[\hbar / 2 m \tilde{\Omega}]^{1 / 2}$. For the lattice setup at $\alpha=1 / 2$, the two components are replaced by the two "layers", and the effective filling factor of this state is defined with respect to $\tilde{\Omega}$ and given by $\tilde{\nu} \equiv \hbar \varrho \pi / m \tilde{\Omega}=2 / 3$. The state can be extended to a general $\alpha_{c}=l / n$ with $\tilde{\nu}=n /(n+1)$.

Since this state has exactly zero interaction energy, it would be the lowest step of the density profile in a slowly varying external potential. Adding more particles to the system leads to a trade-off between an increasing potential energy when the particle is added to the "edge" of the 221 state and an increased interaction energy if the particle is added at the center of the trap where the potential energy is lowest. This eventually leads to a stepped density profile. So far it was not possible to determine with certainty what the next higher step looks like, but it might be either a $\tilde{\nu}=1$ Read-Rezayi [1] state or a $\tilde{\nu}=4 / 3$ non-Abelian spin singlet (NASS) state 68. Depending on the energies of the states, which are not exactly known, also both steps can occur. Higher states might be the Read-Rezayi states with $\tilde{\nu}=3 / 2, \tilde{\nu}=2$, and so on, eventually leading to a vortex lattice [69].

It is still unknown where exactly the transition to this vortex lattice phase occurs. The Lindemann criterion [69, 70] gives the order of magnitude estimate $\nu \sim 10$ for both one- and two-component systems, whereas numerical studies of the single component system [65] give $\nu \sim 2-6$. We hence conjecture that our two- "layer" system has a similar critical $\tilde{\nu}$. To investigate the vortex lattice at a large filling factor and $\alpha$ close to $1 / 2$, we have done numerical simulations using imaginary time propagation of the Gutzwiller ansatz [7]

$$
|\psi\rangle=\prod_{i, j}\left[\sum_{n=0}^{n_{\max }} c_{i, j, n}\left(\hat{a}_{i, j}^{\dagger}\right)^{n}|\mathrm{vac}\rangle\right],
$$

where the state is specified by the complex numbers $c_{i, j, n}$. This ansatz can describe both superfluid vortex lattice and Mott insulator states, but in general not off-site correlated states such as the fractional quantum Hall states.

A typical density distribution of our calculations using a random initial state is shown in Fig. 1(a). It exhibits alternating stripes as have been predicted for a continuum two-component system [72, 73]. However, in our case the two components are not two different species or
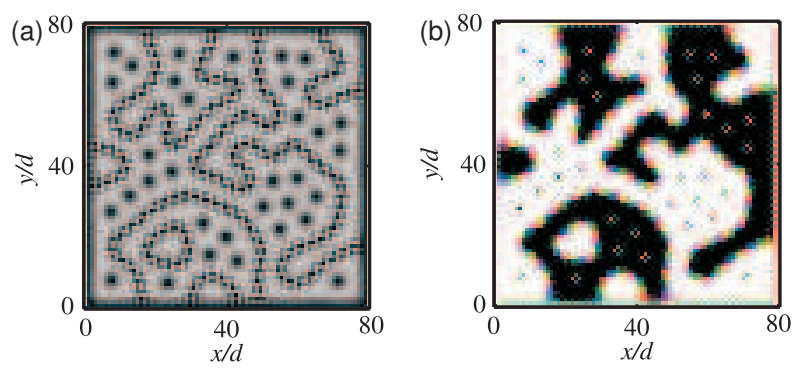

FIG. 1: (Color online) Numerical approximation to the ground state of the $\alpha_{c}=1 / 2$ vortex lattice phase, calculated using imaginary time propagation of a Gutzwiller ansatz. (a) Density of the atom distribution, black stands for no density, white means high superfluid density, red (hardly visible) means high Mott insulator density. (b) Population of the two "layers", black and white depict the two components, color encodes the phase of the superposition of those two components. The parameters are $\alpha=0.48, U=0.1 J$, and $\tilde{\nu}=21.2$.

states of one species, but represent the two "layers" corresponding to the two components of the wave function. For a better distinction these two components are plotted with different colors in Fig. 1(b). A direct measurement of these two components analogous to the measurement of two different species is not possible. Instead, to make the two components visible we calculated the overlaps of the small scale solutions $\mathbf{v}^{(k)}$ with groups of lattice sites of the Gutzwiller solution. These overlaps correspond to the large-scale functions $\chi_{k}$ at the respective lattice sites. Our calculations show that the densities described by the two components indeed add up to the total density of the system and confirm that at least for the chosen parameters the two-component description is meaningful. We find that the stripes straighten out only very slowly with increasing imaginary time (or decreasing temperature), similar to the findings in [73], with a slight tendency to phase separation. The perfectly straight stripes observed in [72] exist only in the zero temperature limit. At exactly $\alpha=\alpha_{c}=l / n$, we have $\tilde{\nu}=\infty$ and one has an $n$ component superfluid with no vortices. This may be the possible superfluid phases found at $\alpha=1 / 3,1 / 2$ in the exact diagonalizations of Sørensen and coworkers [36].

\section{PROBING THE STATES}

After having demonstrated that a very wide range of different states can occur within our setup, the question arises how these states can be distinguished from each other. Fractional quantum Hall states for which Eq. (11) is valid are characterized by a fixed $\tilde{\nu}$, and hence the density for these states will obey the condition $\varrho \propto\left|\alpha-\alpha_{c}\right|$. For "ordinary" fractional quantum Hall states with $\alpha \ll 1$ the characteristic parameter is $\nu$, which consequently leads to a density that obeys $\varrho \propto \alpha$ as discussed in Sec. [II] In contrast, for Mott insulating states, the density is fixed to integer filling and does 
neither depend on $\alpha$ nor on $\alpha-\alpha_{c}$. An in-trap density profile measurement could hence distinguish these states by comparing measurements at different artificial magnetic fields (and thus different $\alpha$ ), as well as measuring the ratio $\nu$ (or $\tilde{\nu}$ ), at which these incompressible states occur.

However, a more clear-cut distinction between the occurring states is possible by using methods such as timeof-flight expansions, and measuring noise correlations or mass currents. In the following sections we show that they reveal additional information which helps to identify the respective states. We should note that some of our results rely on Monte-Carlo simulations of small systems or rough estimates of particle energies and thus they should be considered as qualitative estimates only.

\section{A. Time-of-flight expansion}

The time-of-flight expansion is a standard measurement tool for ultracold atomic systems. All potentials (including the artificial magnetic field) are turned off instantaneously and the atomic cloud is allowed to expand freely for a certain time before measuring its density distribution. Since for an optical lattice setup interactions during the expansion can be neglected and the time can be chosen long enough such that the cloud expands to several times its initial size the result of this process is 74

$$
\varrho_{m}(\mathbf{X})=\left|\tilde{w}\left(\mathbf{X} m_{0} / \hbar t\right)\right|^{2} \sum_{\mathbf{x}_{1}, \mathbf{x}_{2}} \varrho_{1}\left(\mathbf{x}_{1} ; \mathbf{x}_{2}\right) \mathrm{e}^{\mathrm{i} \mathbf{X} \cdot\left(\mathbf{x}_{1}-\mathbf{x}_{2}\right) m_{0} / \hbar t}
$$

Here $\tilde{w}$ is the Fourier transform of the Wannier function describing an atom in a single lattice site, $\varrho_{1}$ is the one-particle density matrix, the sum is taken over all lattice site vectors $\mathbf{x}_{i}, \varrho_{m}(\mathbf{X})$ is the measured density at position $\mathbf{X}$ and time $t$ after release, and $m_{0}$ is the free mass. Note that the "momentum" measured by this process is the free space momentum $-i \hbar \nabla$. It is not to be confused with the momentum of the system Hamiltonian $\left(-i \hbar \partial_{x}-2 m \Omega y,-i \hbar \partial_{y}\right)$. Furthermore, the operators $\hat{a}_{j}$ need to be transformed into the laboratory frame, and $\varrho_{1}$ is expressed in terms of these laboratory frame operators. This is because the sudden switch-off of the artificial magnetic field does not obey Maxwell's equations, which breaks gauge invariance.

As shown in [75], for a low $\alpha$ one can calculate the single particle density matrix $\varrho_{1}$ of the constant density lowest Landau level states, which include the Laughlin, Read-Rezayi, 221, and NASS states. For large $\alpha$, similar expressions with an additional factor $\sum_{k} \mathbf{v}^{(k) *} \mathbf{v}^{(k)}$ from the small scale structure apply. This gives for the density after the release

$$
\begin{aligned}
\varrho_{m}(\mathbf{X})=\mid \tilde{w} & \left.\left(\mathbf{X} m_{0} / \hbar t\right)\right|^{2} \sum_{i, j} \sum_{k} \mathbf{v}^{\prime(k) *}(i) \mathbf{v}^{\prime(k)}(j) \frac{\tilde{\nu}}{2 \pi n r_{0}^{2}} \\
& \times \exp \left\{-\frac{\left(\mathbf{x}_{i}-\mathbf{x}_{j}\right)^{2}}{4 r_{0}^{2}}\right. \\
& +\mathrm{i}\left(\frac{X m_{0}}{\hbar t}+\frac{y_{i}+y_{j}}{4 r_{0}^{2}}\right)\left(x_{i}-x_{j}\right) \\
& \left.+\mathrm{i}\left(\frac{Y m_{0}}{\hbar t}-\frac{x_{i}+x_{j}}{4 r_{0}^{2}}\right)\left(y_{i}-y_{j}\right)\right\},
\end{aligned}
$$

where the vectors $\mathbf{x}_{j}=\left(x_{j}, y_{j}\right)$ label the lattice sites, and the prime indicates that we have taken the $\mathbf{v}^{\prime(k)}$ in symmetric gauge, which doubles their period to $2 n$. Thus the arguments of the $\mathbf{v}^{\prime(k)}$ have to be taken modulus $2 n$.

Summing first over $\mathbf{x}_{1}-\mathbf{x}_{2}$ and approximating this by an integral, we see that each $\mathbf{x}_{1}+\mathbf{x}_{2}$ contributes an approximate Gaussian centered on $\left(-y_{1}-y_{2}, x_{1}+\right.$ $\left.x_{2}\right) \hbar t / 4 m_{0} r_{0}^{2}$, and then summing over $\mathbf{x}_{1}+\mathbf{x}_{2}$ we get the in-trap density profile smeared out on a scale $r_{0}$, rotated through a right angle and scaled by $\hbar t / 4 m_{0} r_{0}^{2}$. Physically this happens because lowest Landau level wavefunctions are very similar to rigid body rotation, and once released from the field the atoms fly apart as if they were rotating. Because the sum is in fact a discrete one, the pattern repeats every reciprocal lattice cell (size $\left.2 \pi \hbar t / d m_{0}\right)$ with a decaying envelope $\tilde{w}$, and high $\alpha$ states can have multiple peaks per reciprocal lattice cell from the small scale structure.

Fig. 2 shows some numerically calculated examples, the pattern repeats every reciprocal lattice cell with a slow decay given by $\tilde{w}[76]$. As expected from the above, fractional quantum Hall states have stepped peaks, further distinguishing them from the featureless $\tilde{w}$ of a Mott insulator [60], while vortex lattice states have ThomasFermi (inverted parabola) peaks. Low $\alpha$ states have one peak per reciprocal lattice cell while $\alpha \approx 1 / 2$ states have 4. The extra peaks (previously described for the vortex lattice in [32, 33]) confirm the existence of a small scale structure. However, they do not show whether one or more "layers" are occupied for multi- "layer" systems.

As a time-of-flight expansion only measures single particle properties, it cannot detect correlations, so different fractional quantum Hall states are distinguished only by their density. In the next section, we will consider an enhancement of this method that can measure the correlations of the states as well.

\section{B. Correlation measurement}

The two-particle correlations of a quantum state manifest themselves in shot noise correlations of the density distribution after a time-of-flight measurement [63, 77], as was recently demonstrated experimentally for the 

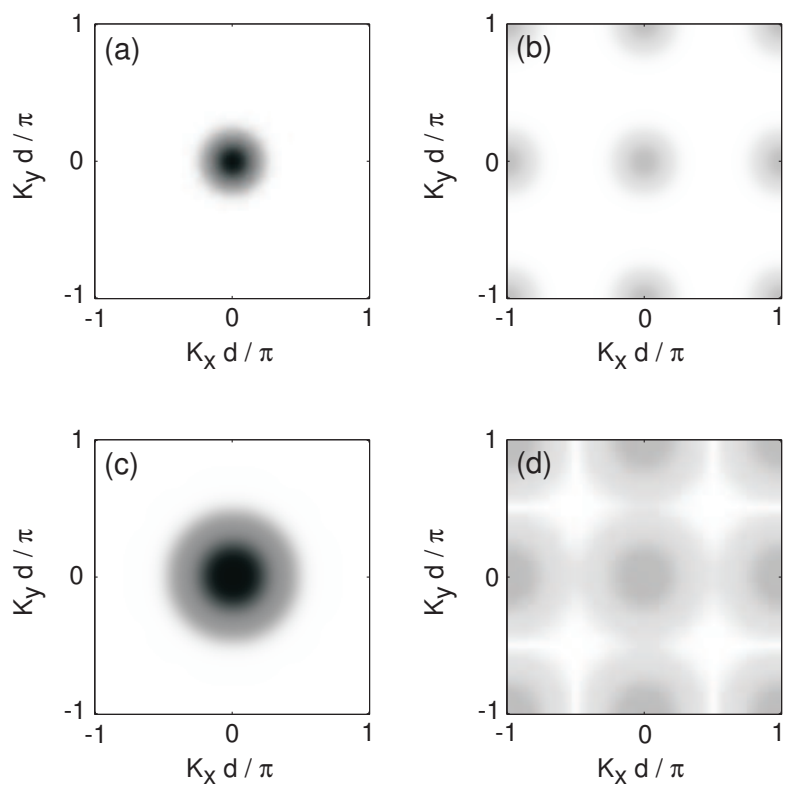

FIG. 2: Numerically calculated time-of-flight expansions in symmetric gauge of $(\mathrm{a}, \mathrm{c})$ a Laughlin and Read-Rezayi state $(\nu=1 / 2,1)$, and $(\mathrm{b}, \mathrm{d})$ a 221 and NASS state $(\tilde{\nu}=2 / 3,4 / 3)$. The initial states are circular with diameter 100 lattice sites for the outer ring and 50 for the inner one. The artificial magnetic field is chosen such that (a) $\alpha=0.005$, (b) $\alpha=$ 0.505 , (c) $\alpha=0.01$, and (d) $\alpha=0.51$. The resulting larger fields $\Omega$ or $\tilde{\Omega}$ in (c,d), respectively, lead to a wider expansion of the cloud. The shown expansions do not include the $\tilde{w}$ slow decay, as the speed of this is implementation dependent. A darker color corresponds to a higher density of particles, and figures (a) and (b) are to the same brightness scale, as are (c) and (d). Shown is the first Brillouin zone, and $\mathbf{K}=m_{0} \mathbf{X} / \hbar t$.

Mott insulator [60]. At time $t$ after release from an optical lattice these correlations are given by [63]

$$
\begin{aligned}
& G\left(\mathbf{r}, \mathbf{r}^{\prime}\right) \propto t^{-6} \sum_{i i^{\prime} j j^{\prime}} \mathrm{e}^{\mathrm{i} \mathbf{R}_{i i^{\prime}} \cdot \mathbf{Q}(\mathbf{r})+\mathrm{i} \mathbf{R}_{j j^{\prime}} \cdot \mathbf{Q}\left(\mathbf{r}^{\prime}\right)}\left\langle\hat{a}_{i}^{\dagger} \hat{a}_{j}^{\dagger} \hat{a}_{j^{\prime}} \hat{a}_{i^{\prime}}\right\rangle \\
&-\langle\hat{n}(\mathbf{r})\rangle\left\langle\hat{n}\left(\mathbf{r}^{\prime}\right)\right\rangle,
\end{aligned}
$$

where $i, j, i^{\prime}, j^{\prime}$ run over all lattice sites, $\mathbf{R}_{i i^{\prime}}$ is the displacement vector from site $i$ to site $i^{\prime}$, and $\mathbf{Q}(\mathbf{r})=m_{0} \mathbf{r} / \hbar t$ with $m_{0}$ the free mass. For states described by a continuum wavefunction the expectation values over the quantum operators are given by $\left\langle\hat{a}_{i}^{\dagger} \hat{a}_{j}^{\dagger} \hat{a}_{i^{\prime}} \hat{a}_{j^{\prime}}\right\rangle=d^{2} \varrho_{2}\left(i, j ; i^{\prime}, j^{\prime}\right)$. Here, $\varrho_{2}\left(i, j ; i^{\prime}, j^{\prime}\right)$ is the continuum two-particle density matrix, which for lowest Landau level states with constant density is of the form 75]

$$
\begin{aligned}
\varrho_{2}\left(i, j ; i^{\prime}, j^{\prime}\right)= & \mathrm{e}^{-\frac{1}{4}\left[\left|z_{i}\right|^{2}+\left|z_{i^{\prime}}\right|^{2}+\left|z_{j}\right|^{2}+\left|z_{j^{\prime}}\right|^{2}-2\left(z_{i}^{*} z_{i^{\prime}}+z_{j}^{*} z_{j^{\prime}}\right)\right]} \\
& \times\left(\frac{\tilde{\nu}}{2 \pi n r_{0}^{2}}\right)^{2} g\left(\left(z_{i}-z_{j}\right)^{*}\left(z_{i^{\prime}}-z_{j^{\prime}}\right)\right) .
\end{aligned}
$$

Note that $\tilde{\nu}=\nu$ and $n=1$ for $\alpha_{c}=0$. The function $g(z)$ for arbitrary complex $z$ is derived by analytic con-

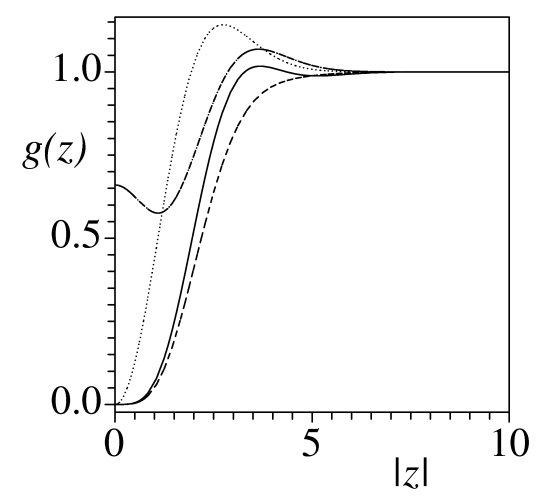

FIG. 3: Two-point functions for continuum fractional quantum Hall states: $\nu=1 / 2$ Laughlin state (solid), $\tilde{\nu}=2 / 3221$ state with $g_{11}=g_{22}$ (dashed) and $g_{12}=g_{21}$ (dotted), and $\nu=1$ Read-Rezayi state (dash-dotted).

tinuation of the ordinary two-point correlation function $g\left(\left|z_{i}-z_{j}\right|^{2}\right)$. If the behavior of a two-component state close to $\alpha=1 / 2$ is to be investigated, we need to replace

$$
\begin{aligned}
\left\langle\hat{a}_{i}^{\dagger} \hat{a}_{j}^{\dagger} \hat{a}_{i^{\prime}} \hat{a}_{j^{\prime}}\right\rangle=d^{2} \sum_{k_{1}, k_{2}} \varrho_{2}^{\left(k_{1}, k_{2}\right)}\left(z_{i}, z_{j} ; z_{i^{\prime}}, z_{j^{\prime}}\right) & \\
& \times \mathbf{v}^{\left(k_{1}\right) *}(i) \mathbf{v}^{\left(k_{1}\right)}\left(i^{\prime}\right) \mathbf{v}^{\left(k_{2}\right) *}(j) \mathbf{v}^{\left(k_{2}\right)}\left(j^{\prime}\right),
\end{aligned}
$$

where $\varrho_{2}^{(1,1)}=\varrho_{2}^{(2,2)}$ is the continuum density matrix for two particles of the same type and $\varrho_{2}^{(1,2)}=\varrho_{2}^{(2,1)}$ for two particles of opposite type. The density matrices $\varrho_{2}^{\left(k_{1}, k_{2}\right)}$ are described by expressions analogous to Eq. (17), where the function $g\left(|z|^{2}\right)$ is replaced by $g_{k_{1}, k_{2}}\left(|z|^{2}\right)$. To obtain $g_{k_{1}, k_{2}}$ we fit the series

$$
\begin{aligned}
g_{11}\left(|z|^{2}\right) & =g_{22}\left(|z|^{2}\right) \\
= & 1+\mathrm{e}^{-|z|^{2} / 2}+\sum_{m \geq 0 \text { even }} \frac{2 c_{m}}{m !}\left(\frac{|z|^{2}}{4}\right)^{m} \mathrm{e}^{-|z|^{2} / 4}, \\
g_{12}\left(|z|^{2}\right) & =g_{21}\left(|z|^{2}\right)=1+\sum_{m \geq 0} \frac{2 \tilde{c}_{m}}{m !}\left(\frac{|z|^{2}}{4}\right)^{m} \mathrm{e}^{-|z|^{2} / 4},
\end{aligned}
$$

to Monte Carlo data for the respective state. This works for any lowest Landau level state as shown in [78, 79], where we take a sign change into account because our particles are bosons. Our calculations show that the Laughlin and 221 states have $g(0)=0$ (see Fig. (3), meaning that particles cannot come together and the interaction energy is hence zero, while the NASS and ReadRezayi states have $g(0)>0$ and hence non-zero interaction energy (the NASS state is not shown since it is qualitatively similar to the Read-Rezayi state).

Eq. (16) involves a sum over four complex variables, which makes its direct numerical evaluation computa- 
tionally intensive. To simplify this problem, we integrate over $\mathbf{r}+\mathbf{r}^{\prime}$, which gives a delta function setting $z_{i}+z_{j}-z_{i^{\prime}}-z_{j^{\prime}}=0$, and removes all explicit dependence on $z_{i}+z_{j}+z_{i^{\prime}}+z_{j^{\prime}}$. The resulting correlation, which (ignoring the $t^{-6}$ prefactor) is a function of momentum difference $\Delta \mathbf{k}=\mathbf{Q}(\mathbf{r})-\mathbf{Q}\left(\mathbf{r}^{\prime}\right)$, can hence be evaluated for an infinitely extended state by summing over just two complex variables, $z_{i}-z_{j}$ and $z_{i^{\prime}}-z_{j^{\prime}}$. However, this does not work for a finite size state because the ranges of $z_{i}-z_{j}$ and $z_{i^{\prime}}-z_{j^{\prime}}$ then do depend on $z_{i}+z_{j}+z_{i^{\prime}}+z_{j^{\prime}}$, and assuming an infinite size state while necessarily summing over a finite range introduces the possibility of numerical artifacts.

Some numerically calculated examples of the noise correlations are shown in Fig. 4, where again one reciprocal lattice cell is shown and the pattern repeats. Fully Bose condensed states, including ideal vortex lattices, have zero correlation. The Laughlin and 221 states are found to show near $100 \%$ anticorrelation at small $\Delta k$. Here we should stress that as this measurement works in Fourier space, this anticorrelation is not the case for all fully real-space anticorrelated states: the Mott insulator, for example, shows positive correlation [60]. Higher density fractional quantum Hall states also show anticorrelation but of reduced strength. Our data suggests that Read-Rezayi states have a ringed pattern with their strongest anticorrelation $(\approx 50 \%$ for $\nu=1$ and $\approx 35 \%$ for $\nu=3 / 2$ ) at a nonzero $\Delta k$, while the $\tilde{\nu}=4 / 3$ NASS state has $\approx 40 \%$ anticorrelation at zero $\Delta k$ and no ring. The presence or absence of this ring might be used to distinguish between the Read-Rezayi and NASS states. Apart from this we observe that the small-scale structure for $\alpha \approx 1 / 2$ is also visible in the noise-correlations, see Figs. 4(d)-4(f): Additional anticorrelation dips at $\Delta k=( \pm \pi / 2 d, \pm \pi / 2 d),( \pm \pi / 2 d, 0),(0, \pm \pi / 2 d)$ occur, which have a similar structure to the central ones at $\Delta k=(0,0)$.

\section{Hall current and disorder}

So far, we discussed only the static properties of the fractional quantum Hall states and how to detect them. However, in solid state physics one of the most important observables is a dynamical property, namely the Hall current. In this section, we will therefore study the atomic case subject to a linear tilt and to disorder.

When a linear potential $V(x, y)=$ may is applied to a continuum fractional quantum Hall system, all states acquire a velocity $a /(2 \Omega)$ at right angles to the potential gradient causing the Hall current. In a perfect continuum system this is an exact result, unaffected by interaction, because such an acceleration term is exactly canceled out by Galilean transforming Eq. (7) to a reference frame moving with this velocity. In a lattice system the result is valid at sufficiently low $\alpha$ for the continuum approximation to apply; at higher $\alpha$ the lattice, which defines a rest frame, becomes important and a different veloc-
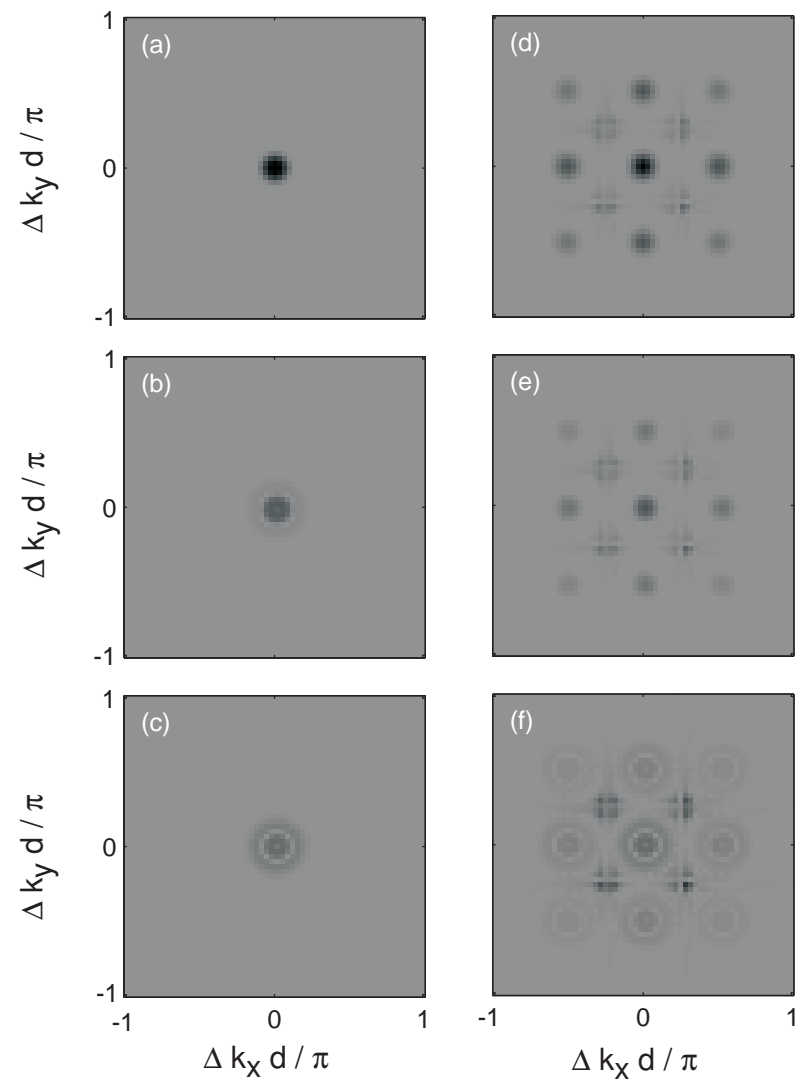

FIG. 4: Numerically calculated shot noise correlations for an infinite system in symmetric gauge. The states are (a) Laughlin, (b) $\nu=1$ Read-Rezayi, (c) $\nu=3 / 2$ Read-Rezayi, (d) 221, (e) $\tilde{\nu}=4 / 3$ NASS, and (f) $\tilde{\nu}=3 / 2$ Read-Rezayi. The artificial magnetic field was chosen to give $\alpha=0.01$ in (a)-(c), and $\alpha=0.51$ in (d)-(f). Gray corresponds to no correlation, whereas black corresponds to $100 \%$ anticorrelation. We cannot rule out that the four dips at $\Delta k=( \pm \pi / 4 d, \pm \pi / 4 d)$ are artifacts arising from our approximations.

ity can occur [9]. In particular, near simple rational $\alpha$ where Eq. (11) is valid, the velocity is $a /(2 \tilde{\Omega})$, which can be very different: for $\alpha<\alpha_{c}$ it even has the opposite sign, meaning that the current flows in the opposite direction. A negative Hall current was also observed for a single particle in a lattice using Kubo response theory [49].

For nonlinear potentials of large length scale compared to the magnetic length $r_{0}$, the single particle eigenstates lie along the equipotential lines of the potential and the Hall current flows along those lines at the velocity given by the local potential gradient. This motion is not visible in equilibrium as the steps in the density profile lie along equipotentials as well. However, it can be made visible by putting the system out of equilibrium, for example, by suddenly changing the trapping potential. Relaxation to equilibrium will be slow because nonforward scattering is energetically forbidden in the fractional quantum Hall system, making the Hall current a supercurrent [80, 81].

It has been shown that when sufficiently mild disorder 
is added to a fractional quantum Hall system, some of the particles become localized and cannot carry current, but those which remain free move faster and the average velocity is still $a /(2 \tilde{\Omega})[80,81]$. For example, for smooth disorder $V(x, y)=$ may $+V_{r}(x, y)$, where $V_{r}$ is a random potential with length scale much larger than $r_{0}$ and zero average, the expression for the $x$ direction of the velocity, $v_{x}=(\partial V / \partial y) /(2 m \tilde{\Omega})$, remains valid. Hence particles on closed equipotentials are confined to those lines, but the random part of $v_{x}$ averages to zero so the average velocity in the $x$ direction remains $a /(2 \tilde{\Omega})$. The current is hence determined by the width over which each extended level is occupied. A simple model of this is to describe the disorder by a density of states $\varrho_{d}(E)$, given by the number of lowest Landau level states per unit energy interval and per unit area. This gives $\int \varrho_{d}(E) \mathrm{d} E=m \tilde{\Omega} /(\pi \hbar)$. In the case of smooth disorder, $\varrho_{d}(E)$ is proportional to the probability distribution of the noise potential. For a linear geometry, we then have

$$
\begin{aligned}
& N=L \int \mathrm{d} y \int^{\mu-V_{1}(y)} \mathrm{d} E \sum_{j \geq 0}\left(\nu_{j}-\nu_{j-1}\right) \varrho_{d}\left(E-\mu_{j}\right), \quad(21) \\
& I=\frac{m}{2 \pi \hbar} \int \mathrm{d} y \frac{d V_{2}}{d y} \int^{\mu-V_{1}(y)} \mathrm{d} E \sum_{j \geq 0}\left(\nu_{j}-\nu_{j-1}\right) \delta\left(E-\mu_{j}\right),
\end{aligned}
$$

where $L$ is the length of the system along the $x$ axis, $I$ is the net current in the $x$ direction, $N$ the number of particles, and $\mu$ the chemical potential. The fractional quantum Hall states are taken at filling factors $\nu_{j}$ and chemical potential $\mu_{j}$, and $V$ is suddenly changed from $V_{1}(y)$ to $V_{2}(y)$ to achieve the nonequilibrium situation. The exact shape of the disorder and thus of $V_{1}(y)$ and $V_{2}(y)$ are not important for our qualitative investigations. These equations are valid for weak trapping potentials, where $d V / d y$ is much smaller than the disorder term. Stronger potentials can break weakly localized states free, replacing the $\delta\left(E-\mu_{j}\right)$ density of extended states in $I$ by a finite width distribution.

In a square-well potential (as in a solid state system) the localized states create a finite range of filling factors over which a given number of fractional quantum Hall extended state levels are completely full, giving rise to the almost perfectly flat fractional quantum Hall plateaus. In a harmonic trap there will not be fractional quantum Hall plateaus, only corners each time a new extended level begins to fill, see Fig. [5. Between those corners, there exist different fractional quantum Hall states. Unlike the square-well case, it is possible to obtain the complete distribution $\varrho_{d}(E)$ by measuring $I$ against $N($ or $\tilde{\Omega})$.

\section{CONCLUSIONS}

In our work we have studied the low and high field fractional quantum Hall effect in an optical lattice. For

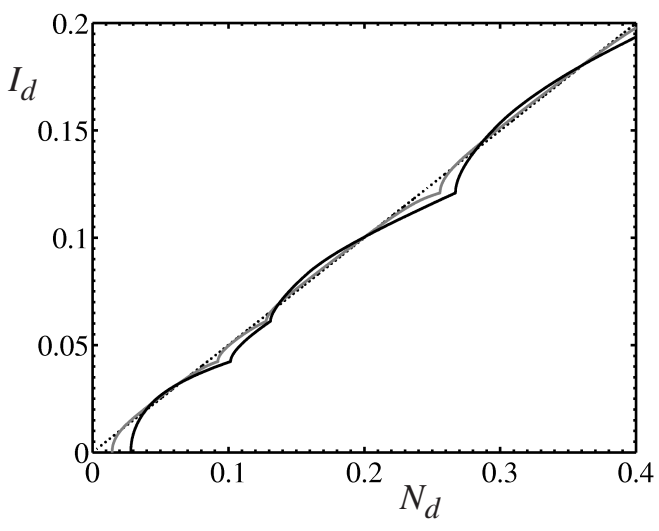

FIG. 5: Dimensionless Hall current $I_{d}=$ $I /\left(2 a m^{2} \sqrt{2 u \tilde{\Omega} / \hbar^{3}} / \omega\right)$ against dimensionless number of atoms per unit length $N_{d}=N /\left(2 L m \sqrt{2 u \tilde{\Omega}^{3} / \hbar^{3}} / \omega\right)$, where $L$ is the length of the system, which is contained in a $1 \mathrm{D}$ harmonic trap $V(x, y)=m \omega^{2} y^{2} / 2+y m a$ at $\alpha \approx 1 / 2$. The linear term yma is turned on after the atoms have come to equilibrium in the trap, and the curves are for no disorder (dotted straight line), maximal disorder (constant density of states, black curve), and Lorentzian disorder of width (1/5)um $\tilde{\Omega} / 2 \pi \hbar$ (gray curve). The corresponding plot for small $\alpha$ is qualitatively similar [9] .

small $\alpha$, corresponding to low field strengths, the continuum approximation can be employed to find the solutions of the Hamiltonian. For high fields corresponding to values of $\alpha$ close to simple rationals, $\alpha \approx \alpha_{c}=l / n$, we showed that the states can be approximated by an $n$-component wave function, where each component is described by a slowly varying, continuous function $\chi^{(k)}$. These functions are solutions of a differential equation similar to the $\alpha \ll 1$ case, but with an artificial magnetic field term $\tilde{\Omega}=\hbar \pi\left(\alpha-\alpha_{c}\right) / m d^{2}$ and an effective filling factor $\tilde{\nu} \equiv \hbar \varrho \pi / m \tilde{\Omega}$ instead of the usual $\Omega$ and $\nu$.

We have shown that many interesting topological states may occur in our setup, even if it is subject to a weak harmonic trapping potential in one direction. These states include the Laughlin, the Read-Rezayi, the 221, and the non-Abelian spin singlet (NASS) state. Numerical investigations also showed the occurrence of a striped vortex lattice phase close to $\alpha \approx 1 / 2$, as predicted for a two-component system at low $\alpha$. In our case the two components did not correspond to two different atomic species, but to the two components $\chi^{(k)}$ of the wave function.

We have furthermore demonstrated that the above states can be distinguished by suitable measurements. Simple time-of-flight expansions reveal additional structures for higher $\alpha$ states, but do not necessarily allow for distinguishing between the different quantum Hall states. More insight can be gained by measuring twopoint correlation functions. For the Laughlin state, this function is zero for $\left|z_{1}-z_{2}\right|=0$, meaning that there are 
never two atoms at the same place, whereas for the ReadRezayi state with $\nu=1$ the correlation function exhibits a finite value at zero distance. We, moreover, showed that the different states also exhibit different noise correlations, which can be employed to distinguish between them. Again, higher $\alpha$ states show additional structures due to their additional small scale symmetries. Further, our results suggest that the Read-Rezayi states exhibit a ring structure, which allows one to distinguish them from the Laughlin or 221 states.

In analogy to the semiconductor Hall effects, it is possible to displace the optical lattice and measure mass transport effects, which behave similarly to the condensed matter analogues. Especially Hall currents proportional to $\alpha-\alpha_{c}$ rather than $\alpha$ have been predicted, which may lead to a negative sign in the direction of the mass flow.

\section{Acknowledgments}

The authors thank Sarah Al-Assam for helpful comments. This work was supported by the United Kingdom EPSRC through QIP IRC (Grant No. GR/S82176/01) and EuroQUAM Project No. EP/E041612/1, the EU through the STREP project OLAQUI, the Merton College Domus Scholarship (R.N.P.), and the Keble Associ- ation (A.K.).

\section{APPENDIX A: DERIVATION OF HIGH $\alpha$ STATES}

In this appendix we give details on the derivation of the Hamiltonian equation (8) for large $\alpha$ close to a rational $\alpha_{c}=l / n$ with small integers $l$ and $n$. We assume that apart from the optical lattice the atomic gas experiences no trapping potential in the $x$ direction, but might be trapped by a (slowly varying) harmonic potential $V(x, y)=\frac{1}{2} m \omega^{2} y^{2}$ in the $y$-direction. Note that the mass in this potential is the effective mass $m=\hbar / 2 J d^{2}$ introduced earlier. Motivated by previous findings [9], we make for the wave function the ansatz $\psi(n s+i, n r+j)=$ $d \phi_{j}((n r+j) d) \mathrm{e}^{\mathrm{i} K(n s+i) d}$. Here, $i, j=1, \ldots, n$ and $r, s$ are integers, and we define $x=(n s+i) d, y=(n r+j) d$. Since $\hat{H}$ is independent of $x$ if the potential $V$ is, the $x$ quasimomentum $K$ is conserved exactly for our choice of $V$. Let $K=2 \pi k l / n d+\tilde{K}$, where $k$ is an integer and $\tilde{K} \ll 1 / n$ to yield an $n$ site periodicity plus slow variation in the $x$ direction. The action of Hamiltonian equation (11) with $U=0$ gives

$$
\begin{aligned}
\hat{H}|\psi\rangle= & -J d \sum_{r, s} \sum_{i, j}\left[\mathrm{e}^{2 \pi \mathrm{i} \alpha(n r+j)+\mathrm{i} K(n s+i-1) d} \phi_{j}(n r+j)+\mathrm{e}^{-2 \pi \mathrm{i} \alpha(n r+j)+\mathrm{i} K(n s+i+1) d} \phi_{j}(n r+j)\right. \\
& \left.+\phi_{j-1}(n r+j-1) \mathrm{e}^{\mathrm{i} K(n s+i) d}+\phi_{j+1}(n r+j+1) \mathrm{e}^{\mathrm{i} K(n s+i) d}\right] \hat{a}_{n r+j, n s+i}^{\dagger}|\mathrm{vac}\rangle \\
& +\sum_{r, s} \sum_{i, j} V(n r+j, n s+i) \hat{a}_{n r+j, n s+i}^{\dagger}|\mathrm{vac}\rangle .
\end{aligned}
$$

Using the fact that $\phi_{j}$ is only slowly varying for constant index $j[9]$, we can approximate derivatives by appropriate discrete differences and make use of the fact

$$
\frac{\phi_{j \pm 1}(y)}{d^{2}} \pm \frac{1}{d} \frac{\partial \phi_{j \pm 1}(y)}{\partial y}+\frac{1}{2} \frac{\partial^{2} \phi_{j \pm 1}(y)}{\partial y^{2}} \approx \frac{1}{d^{2}} \phi_{j \pm 1}[(n r+j \pm 1) d]
$$

Collecting all terms with the same creation operators in Eq. (A1) we can derive a Hamiltonian for $\phi_{j}$, acting as

$$
\begin{aligned}
\hat{H}_{1} \phi_{j}=- & \frac{\hbar^{2}}{m d^{2}} \cos \left(\frac{2 \pi j l}{n}+\frac{2 m \tilde{\Omega} y d}{\hbar}-K d\right) \phi_{j}+\frac{1}{2} m \omega^{2} y^{2} \phi_{j}-\frac{\hbar^{2}}{2 m}\left(\frac{\phi_{j+1}}{d^{2}}+\frac{1}{d} \frac{\partial \phi_{j+1}}{\partial y}+\frac{1}{2} \frac{\partial^{2} \phi_{j+1}}{\partial y^{2}}\right. \\
& \left.+\frac{\phi_{j-1}}{d^{2}}-\frac{1}{d} \frac{\partial \phi_{j-1}}{\partial y}+\frac{1}{2} \frac{\partial^{2} \phi_{j-1}}{\partial y^{2}}\right)+O(d) .
\end{aligned}
$$


If the condition $2 m \tilde{\Omega} y d / \hbar \ll 1$ holds over the range where the wave function is appreciable, namely $y \sim l_{\chi}$, we can apply the Taylor expansion to the cosine, which after collecting terms in orders of $d$ yields

$$
\begin{aligned}
\hat{H}_{1} \phi_{j}= & -\frac{\hbar^{2}}{2 m d^{2}}\left[\phi_{j+1}+2 \cos \left(\frac{2 \pi(j-k) l}{n}\right) \phi_{j}+\phi_{j-1}\right]-\frac{\hbar^{2}}{2 m d}\left[\left(\tilde{K}-\frac{2 m \tilde{\Omega} y}{\hbar}\right) 2 \sin \left(\frac{2 \pi(j-k) l}{n}\right) \phi_{j}+\frac{\partial \phi_{j+1}}{\partial y}-\frac{\partial \phi_{j-1}}{\partial y}\right] \\
& -\frac{\hbar^{2}}{2 m}\left[-\left(\tilde{K}-\frac{2 m \tilde{\Omega} y}{\hbar}\right)^{2} \cos \left(\frac{2 \pi(j-k) l}{n}\right) \phi_{j}-\frac{m^{2} \omega^{2} y^{2}}{\hbar^{2}} \phi_{j}+\frac{1}{2} \frac{\partial^{2} \phi_{j+1}}{\partial y^{2}}+\frac{1}{2} \frac{\partial^{2} \phi_{j-1}}{\partial y^{2}}\right]+O(d) .
\end{aligned}
$$

Note that for $n=1$ or 2 the odd powers in $d$ cancel by symmetry and we get an expansion in $d^{2}$, but that for larger $n$ the expansion is in $d$. Define $\Phi=\left(\phi_{1} \ldots \phi_{n}\right)^{T}$ and expand in powers of $d: \Phi=\Phi^{(0)}+d \Phi^{(1)}+d^{2} \Phi^{(2)}+O\left(d^{3}\right)$. Similarly expand the energy $E=E_{0} / d^{2}+E_{1} / d+E_{2}+O(d)$. We define the matrix

$$
\mathbf{A}_{0}=\left(\begin{array}{cccccc}
2 \cos 2 \pi(1-k) l / n & 1 & 0 & \cdots & 0 & 1 \\
1 & 2 \cos 2 \pi(2-k) l / n & 1 & & & 0 \\
0 & 1 & \ddots & \ddots & & \vdots \\
\vdots & & \ddots & \ddots & \ddots & 0 \\
0 & & & \ddots & \ddots & 1 \\
1 & 0 & \cdots & 0 & 1 & 2 \cos 2 \pi(n-k) l / n
\end{array}\right)
$$

The $O\left(1 / d^{2}\right)$ terms then become $\mathbf{A}_{0} \Phi^{(0)}=$ $-2 m E_{0} \Phi^{(0)} / \hbar^{2}$, so $\Phi^{(0)}$ is an eigenvector of $\mathbf{A}_{0}$, with the ground state having the largest eigenvalue. Assuming nondegenerate eigenvalues, $\Phi^{(0)}(y)$ is hence proportional to the same (normalized) eigenvector $\mathbf{v}^{(k)}$, and can depend on $y$ only in overall magnitude, i.e., $\Phi^{(0)}(y)=\chi(y) \mathbf{v}^{(k)}$.

Since changing $k$ is equivalent to changing the origin of $j$, the eigenvalues are the same for all $k$, with ground state eigenvectors $v_{j}^{(k)}=v_{j-k}$, where $\mathbf{v}$ is the normalized ground state eigenvector for $k=0$ and the subscript $j-k$ wraps around modulus $n$. This gives $n$ degenerate ground states $k=0, \ldots, n-1$, which are orthogonal because of their different $K$ values. For simplicity we take $k=0$ in the remainder of this derivation.

For the $O(1 / d)$ terms, define

$$
\mathbf{A}_{1}=\left(\begin{array}{ccccc}
0 & 1 & 0 & \cdots & -1 \\
-1 & 0 & 1 & & \vdots \\
0 & -1 & 0 & \ddots & 0 \\
\vdots & & \ddots & \ddots & 1 \\
1 & \cdots & 0 & -1 & 0
\end{array}\right)
$$

$$
\mathbf{A}_{2}=2 \operatorname{diag}[\sin (2 \pi l / n), \sin (4 \pi l / n), \ldots, \sin (2 n \pi l / n)],
$$

where diag denotes a diagonal matrix with the argument as the entries on the diagonal. Define furthermore $\mathbf{w}_{1}=$
$\mathbf{A}_{1} \mathbf{v}, \mathbf{w}_{2}=\mathbf{A}_{2} \mathbf{v}$. This yields

$$
\begin{aligned}
\left(\mathbf{A}_{0}+2 m E_{0} / \hbar^{2}\right) \boldsymbol{\Phi}^{(1)}(y)= & \frac{2 m E_{1}}{\hbar^{2}} \chi(y) \mathbf{v}+\frac{d \chi}{d y} \mathbf{w}_{1} \\
& +\left(K-\frac{2 m \tilde{\Omega} y}{\hbar}\right) \chi(y) \mathbf{w}_{2} .
\end{aligned}
$$

The left hand side of Eq. (A8) is orthogonal to $\mathbf{v}$ because $\mathbf{A}_{0}+2 m E_{0} / \hbar^{2}$ annihilates $\mathbf{v}$ and is Hermitian, while $\mathbf{w}_{1}$ is orthogonal to $\mathbf{v}$ because $\mathbf{A}_{1}$ is antisymmetric and hence so is $\mathbf{v}^{T} \mathbf{A}_{1} \mathbf{v}$, but the latter is a number so it can only be antisymmetric if it is zero. Hence a solution can only exist if $\mathbf{w}_{2}$ is also orthogonal to $\mathbf{v}$ and $E_{1}=0$; this is the case for all $\alpha_{c}=1 / 8,1 / 7,1 / 6,1 / 5,1 / 4,2 / 7,1 / 3,3 / 8,2 / 5,3 / 7,1 / 2$ within numerical accuracy [9], but we have not been able to prove that it is always the case.

For the $O(1)$ terms define

$$
\mathbf{A}_{3}=\left(\begin{array}{ccccc}
0 & 1 & 0 & \cdots & 1 \\
1 & 0 & 1 & & \vdots \\
0 & 1 & 0 & \ddots & 0 \\
\vdots & & \ddots & \ddots & 1 \\
1 & \cdots & 0 & 1 & 0
\end{array}\right),
$$

$\mathbf{A}_{4}=\operatorname{diag}[\cos (2 \pi l / n), \cos (4 \pi l / n), \ldots, \cos (2 n \pi l / n)]$, 
giving

$$
\begin{aligned}
& \left(\frac{2 m E_{2}}{\hbar^{2}}-\frac{m^{2} \omega^{2} y^{2}}{\hbar^{2}}\right) \chi(y) \mathbf{v} \\
& -\left(\frac{2 m \tilde{\Omega} y}{\hbar}-K\right)^{2} \chi(y) \mathbf{A}_{4} \mathbf{v}+\frac{1}{2} \frac{\partial^{2} \chi}{\partial y^{2}} \mathbf{A}_{3} \mathbf{v} \\
& +\left[\left(K-\frac{2 m \tilde{\Omega} y}{\hbar}\right) \mathbf{A}_{2}+\frac{\partial}{\partial y} \mathbf{A}_{1}\right]\left(\mathbf{A}_{0}+\frac{2 m E_{0}}{\hbar^{2}}\right)^{-1} \\
& \quad \times\left[\frac{\partial \chi}{\partial y} \mathbf{w}_{1}+\left(K-\frac{2 m \tilde{\Omega} y}{\hbar}\right) \chi(y) \mathbf{w}_{2}\right] \\
& +\left(\mathbf{A}_{0}+\frac{2 m E_{0}}{\hbar^{2}}\right) \mathbf{\Phi}^{(2)}=\mathbf{0} .
\end{aligned}
$$

We note that for nondegenerate eigenvalues of $\mathbf{A}_{0}$ the expressions $\left(\mathbf{A}_{0}+2 m E_{0} / \hbar^{2}\right)^{-1} \mathbf{w}_{j}, j=1,2$, are well-defined since both $\mathbf{w}_{1}$ and $\mathbf{w}_{2}$ are orthogonal to $\mathbf{v}$. Taking the scalar product of Eq. A11 with $\mathbf{v}$ gives a harmonic oscillator equation for $\chi(y)$,

$E_{2} \chi=-\frac{\hbar^{2} C_{1}}{2 m} \frac{\partial^{2} \chi}{\partial y^{2}}+\frac{\hbar^{2} C_{2}}{2 m}\left(\frac{2 m \tilde{\Omega} y}{\hbar}-K\right)^{2} \chi+V(x, y) \chi$

where the dimensionless constants $C_{1}=\mathbf{v}^{T} \mathbf{A}_{3} \mathbf{v} / 2-$ $\mathbf{w}_{1}^{T}\left(\mathbf{A}_{0}+2 m E_{0} / \hbar^{2}\right)^{-1} \mathbf{w}_{1}$ and $C_{2}=\mathbf{v}^{T} \mathbf{A}_{4} \mathbf{v}-\mathbf{w}_{2}^{T}\left(\mathbf{A}_{0}+\right.$ $\left.2 m E_{0} / \hbar^{2}\right)^{-1} \mathbf{w}_{2}$ depend only on $l$ and $n$, and by changing the roles of $x$ and $y$ by using a gauge transformation one can show that $C_{1}=C_{2} \equiv C$. This oscillator has mass $m / C_{1}$, frequency $\omega_{\text {eff }}=\left(4 C_{1} C_{2} \tilde{\Omega}^{2}+C_{1} \omega^{2}\right)^{1 / 2}$, and center $y_{c}=2 \hbar C_{2} K \tilde{\Omega} /\left(4 C_{2} m \tilde{\Omega}^{2}+m \omega^{2}\right)$.
[1] K. v. Klitzing, G. Dorda, and M. Pepper, Phys. Rev. Lett. 45, 494 (1980).

[2] D. C. Tsui, H. L. Stormer, and A. C. Gossard, Phys. Rev. Lett. 48, 1559 (1982).

[3] D. C. Tsui, H. L. Störmer, and A. C. Gossard, Phys. Rev. B 25, 1405 (1982).

[4] A. Y. Kitaev, Ann. Phys. (NY) 303, 2 (2003).

[5] N. R. Gregory Moore, Nucl. Phys. B 360, 362 (1991).

[6] S. Bravyi, Phys. Rev. A 73, 042313 (2006).

[7] S. Das Sarma, M. Freedman, C. Nayak, S. H. Simon, and A. Stern, arXiv:0707.1889v1 (2007).

[8] G. Murthy and R. Shankar, Rev. Mod. Phys. 75, 1101 (2003).

[9] R. N. Palmer and D. Jaksch, Phys. Rev. Lett. 96, 180407 (2006).

[10] R. B. Laughlin, Phys. Rev. Lett. 50, 1395 (1983).

[11] N. Read and E. Rezayi, Phys. Rev. B 59, 8084 (1999).

[12] M. Lewenstein, A. Sanpera, V. Ahufinger, B. Damski, A. Sen, and U. Sen, Advances in Physics 56, 243 (2007).

[13] D. Jaksch, C. Bruder, J. I. Cirac, C. W. Gardiner, and P. Zoller, Phys. Rev. Lett. 81, 3108 (1998).

[14] D. Jaksch and P. Zoller, Ann. Phys. (NY) 315, 52 (2005).

[15] A. Sørensen and K. Mølmer, Phys. Rev. Lett. 83, 2274 (1999).

[16] L.-M. Duan, E. Demler, and M. D. Lukin, Phys. Rev. Lett. 91, 090402 (2003).

[17] W. Hofstetter, J. I. Cirac, P. Zoller, E. Demler, and M. D. Lukin, Phys. Rev. Lett. 89, 220407 (2002).

[18] A. Klein and D. Jaksch, Phys. Rev. A 73, 053613 (2006).

[19] M. Bruderer, A. Klein, S. R. Clark, and D. Jaksch, Phys. Rev. A 76, 011605(R) (2007).

[20] A. Klein, M. Bruderer, S. R. Clark, and D. Jaksch, New J. Phys. 9, 411 (2007).

[21] M. Bruderer, A. Klein, S. R. Clark, and D. Jaksch, New J. Phys. 10, 033015 (2008).

[22] L. Mathey, D.-W. Wang, W. Hofstetter, M. D. Lukin, and E. Demler, Phys. Rev. Lett. 93, 120404 (2004).

[23] M. Lewenstein, L. Santos, M. A. Baranov, and
H. Fehrmann, Phys. Rev. Lett. 92, 050401 (2004).

[24] A. Mering and M. Fleischhauer, Phys. Rev. A 77, 023601 (2008).

[25] H. P. Büchler and G. Blatter, Phys. Rev. Lett. 91, 130404 (2003).

[26] M. Popp, J.-J. Garcia-Ripoll, K. G. Vollbrecht, and J. I. Cirac, Phys. Rev. A 74, 013622 (2006).

[27] M. Popp, J.-J. Garcia-Ripoll, K. G. H. Vollbrecht, and J. I. Cirac, New J. Phys. 8, 164 (2006).

[28] A. Griessner, A. J. Daley, S. R. Clark, D. Jaksch, and P. Zoller, Phys. Rev. Lett. 97, 220403 (2006).

[29] A. Griessner, A. J. Daley, S. R. Clark, D. Jaksch, and P. Zoller, New J. Phys. 9, 44 (2007).

[30] N. R. Cooper, N. K. Wilkin, and J. M. F. Gunn, Phys. Rev. Lett. 87, 120405 (2001).

[31] B. Paredes, P. Fedichev, J. I. Cirac, and P. Zoller, Phys. Rev. Lett. 87, 010402 (2001).

[32] M. Polini, R. Fazio, A. H. MacDonald, and M. P. Tosi, Phys. Rev. Lett. 95, 010401 (2005).

[33] M. Polini, R. Fazio, M. P. Tosi, J. Sinova, and A. H. MacDonald, Laser Physics 14, 603 (2004).

[34] D. Jaksch and P. Zoller, New J. Phys. 5, 56 (2003).

[35] E. J. Mueller, Phys. Rev. A 70, 041603(R) (2004).

[36] A. S. Sørensen, E. Demler, and M. D. Lukin, Phys. Rev. Lett. 94, 086803 (2005).

[37] G. Juzeliūnas and P. Öhberg, Phys. Rev. Lett. 93, 033602 (2004).

[38] G. Juzeliūnas, P. Öhberg, J. Ruseckas, and A. Klein, Phys. Rev. A 71, 053614 (2005).

[39] G. Juzeliūnas, J. Ruseckas, P. Öhberg, and M. Fleischhauer, Phys. Rev. A 73, 025602 (2006).

[40] K. Osterloh, M. Baig, L. Santos, P. Zoller, and M. Lewenstein, Phys. Rev. Lett. 95, 010403 (2005).

[41] J. Ruseckas, G. Juzeliūnas, P. Öhberg, and M. Fleischhauer, Phys. Rev. Lett. 95, 010404 (2005).

[42] N. Goldman, A. Kubasiak, P. Gaspard, and M. Lewenstein, arXiv:0712.2571v2 (2007).

[43] A. Jacob, P. Öhberg, G. Juzeliūnas, and L. Santos, Appl. 
Phys. B 89, 439 (2007).

[44] D. R. Hofstadter, Phys. Rev. B 14, 2239 (1976).

[45] M. C. Geisler, J. H. Smet, V. Umansky, K. von Klitzing, B. Naundorf, R. Ketzmerick, and H. Schweizer, Phys. Rev. Lett. 92, 256801 (2004).

[46] R. O. Umucalllar and M. Ö. Oktel, Phys. Rev. A 76, 055601 (2007).

[47] T.-L. Ho and E. J. Mueller, Phys. Rev. Lett. 89, 050401 (2002).

[48] M. Popp, B. Paredes, and J. I. Cirac, Phys. Rev. A 70, 053612 (2004).

[49] R. Bhat, M. Krämer, J. Cooper, and M. J. Holland, Phys. Rev. A 76, 043601 (2007).

[50] M. Hafezi, A. S. Sørensen, E. Demler, and M. D. Lukin, Phys. Rev. A 76, 023613 (2007).

[51] M. Hafezi, A. S. Sørensen, M. D. Lukin, and E. Demler, Europhys. Lett. 81, 10005 (2008).

[52] V. Bretin, S. Stock, Y. Seurin, and J. Dalibard, Phys. Rev. Lett. 92, 050403 (2004).

[53] V. Schweikhard, I. Coddington, P. Engels, V. P. Mogendorff, and E. A. Cornell, Phys. Rev. Lett. 92, 040404 (2004).

[54] S. Tung, V. Schweikhard, and E. A. Cornell, Phys. Rev. Lett. 97, 240402 (2006).

[55] G. Thalhammer, K. Winkler, F. Lang, S. Schmid, R. Grimm, and J. Hecker Denschlag, Phys. Rev. Lett. 96, 050402 (2006).

[56] A. Widera, F. Gerbier, S. Fölling, T. Gericke, O. Mandel, and I. Bloch, Phys. Rev. Lett. 95, 190405 (2005).

[57] F. Gerbier, A. Widera, S. Fölling, O. Mandel, and I. Bloch, Phys. Rev. A 73, 041602(R) (2006).

[58] A. Öttl, S. Ritter, M. Köhl, and T. Esslinger, Phys. Rev. Lett. 95 95, 090404 (2005).

[59] F. Gerbier, S. Fölling, A. Widera, O. Mandel, and I. Bloch, Phys. Rev. Lett. 96, 090401 (2006).

[60] S. Fölling, F. Gerbier, A. Widera, O. Mandel, T. Gericke, and I. Bloch, Nature 434, 481 (2005).

[61] M. Köhl, H. Moritz, T. Stöferle, C. Schori, and T. Esslinger, J. Low Temp. Phys. 138, 635 (2005).

[62] C. Albrecht, J. H. Smet, K. von Klitzing, D. Weiss,
V. Umansky, and H. Schweizer, Phys. Rev. Lett. 86, 147 (2001).

[63] E. Altman, E. Demler, and M. D. Lukin, Phys. Rev. A 70, 013603 (2004).

[64] A. Klein and M. Fleischhauer, Phys. Rev. A 71, 033605 (2005).

[65] N. R. Cooper, F. J. M. van Lankvelt, J. W. Reijnders, and K. Schoutens, Phys. Rev. A 72, 063622 (2005).

[66] S. Das Sarma and A. Pinczuk, eds., Perspectives in Quantum Hall effects (Wiley, New York, 1997).

[67] Z. F. Ezawa, Quantum Hall Effects: Field Theoretical Approach and Related Topics (World Scientific, Singapore, 2000).

[68] E. Ardonne and K. Schoutens, Phys. Rev. Lett. 82, 5096 (1999).

[69] J. Sinova, C. B. Hanna, and A. H. MacDonald, Phys. Rev. Lett. 89, 030403 (2002).

[70] A. Rozhkov and D. Stroud, Phys. Rev. B 54, R12697 (1996).

[71] W. Krauth, M. Caffarel, and J.-P. Bouchaud, Phys. Rev. B 45, 3137 (1992).

[72] E. J. Mueller and T.-L. Ho, Phys. Rev. Lett. 88, 180403 (2002).

[73] K. Kasamatsu, M. Tsubota, and M. Ueda, Phys. Rev. Lett. 91, 150406 (2003).

[74] I. Bloch, J. Dalibard, and W. Zwerger, arXiv:0704.3011v2 (2007).

[75] A. H. MacDonald and S. M. Girvin, Phys. Rev. B 38, 6295 (1988).

[76] M. Greiner, O. Mandel, T. Esslinger, T. W. Hänsch, and I. Bloch, Nature (London) 415, 39 (2002).

[77] N. Read and N. R. Cooper, Phys. Rev. A 68, 035601 (2003).

[78] S. M. Girvin, Phys. Rev. B 30, 558 (1984).

[79] S. M. Girvin, A. H. MacDonald, and P. M. Platzman, Phys. Rev. B 33, 2481 (1986).

[80] R. E. Prange and S. M. Girvin, The Quantum Hall Effect (Springer Verlag, New York, 1990).

[81] D. R. Yennie, Rev. Mod. Phys. 59, 781 (1987). 